\title{
Diabetes and risk of peripheral artery disease in patients undergoing first-time coronary angiography between 2000 and 2012 - a nationwide study
}

Sadaf Kamil ${ }^{1,2,3^{*}}$, Thomas S. G. Sehested ${ }^{2}$, Nicholas Carlson ${ }^{4,5}$, Kim Houlind ${ }^{6,7}$, Jens F. Lassen ${ }^{8}$, Casper N. Bang ${ }^{9}$, Helena Dominguez ${ }^{2,3}$, Christian T. Pedersen ${ }^{10}$ and Gunnar H. Gislason ${ }^{1,5}$

\begin{abstract}
Background: The risk of peripheral artery disease (PAD) in patients with diabetes mellitus (DM) and coronary artery disease (CAD) is an important and inadequately addressed issue. Our aim is to examine the impact of DM on risk of PAD in patients with different degrees of CAD characterized by coronary angiography (CAG).

Methods: Using nationwide registers we identified all patients aged $\geq 18$ years, undergoing first time CAG between 2000 and 2012. Patients were categorized into DM/Non-DM group, and further classified into categories according to the degree of CAD i.e., no-vessel disease, single-vessel disease, double-vessel disease, triple-vessel disease, and diffuse disease. Risk of PAD was estimated by 5 -year cumulative-incidence and adjusted multivariable Cox-regression models.

Results: We identified 116,491 patients undergoing first-time CAG. Among these, a total of 23.969 (20.58\%) had DM. Cumulative-incidence of PAD among DM patients vs. non-DM were $8.8 \%$ vs. 4.9\% for no-vessel disease, $8.2 \%$ vs. 4.8\% for single-vessel disease, $10.2 \%$ vs. 6.0\% for double-vessel disease, $13.0 \%$ vs. $8.4 \%$ for triple-vessel disease, and $6.8 \%$ vs. $6.1 \%$ for diffuse disease, respectively. For all patients with DM, the cox-regression analysis yielded significantly higher hazards of PAD compared with non-DM patients with HR 1.70 (no-vessel disease), 1.96 (single-vessel disease), 2.35 (double-vessel disease), 2.87 (triple-vessel disease), and 1.46 (diffuse disease), respectively (interaction-p 0.042).

Conclusion: DM appears to be associated with increased risk of PAD in patients with and without established CAD, with increasing risk in more extensive CAD. This observation indicates awareness on PAD risk in patients with DM, especially among patients with advanced CAD.
\end{abstract}

Keywords: Type 2 diabetes mellitus, Peripheral artery disease, Coronary artery disease, Atherosclerosis

\section{Background}

Peripheral artery disease (PAD) is characterized as narrowing of the arteries in the upper and lower extremities arteries [1]. Atherosclerosis is recognized as the most direct and important cause of PAD, leading to acute or chronic limb ischemia [2]. PAD is known to be one of the major complications of diabetes mellitus (DM) [3-6].

\footnotetext{
* Correspondence: sadafkamil88@gmail.com

'Department of Cardiology, Copenhagen University Hospital Herlev-Gentofte, Herlev and Gentofte Hospital, Kildegaardsvej 28, 2900 Hellerup, Denmark ${ }^{2}$ Department of Cardiology, Bispebjerg-Frederiksberg University Hospital, Copenhagen, Denmark

Full list of author information is available at the end of the article
}

The Framingham heart study indicates that 20\% of symptomatic PAD patients presents DM [7]. However, as PAD is mostly asymptomatic, a higher risk of DM in this group of patients is assumed $[8,9]$.

Several large population-based studies have indicated that DM is associated with a two- to four-fold increase in development of PAD compared to non-diabetic patients, moreover studies have shown a three- to fourfold increased risk of mortality in patients with DM and PAD compared to healthy individuals [10-13]. Additionally, patients with concomitant PAD and DM experience reduced quality of life and an increase in long-term

(c) The Author(s). 2019 Open Access This article is distributed under the terms of the Creative Commons Attribution 4.0 International License (http://creativecommons.org/licenses/by/4.0/), which permits unrestricted use, distribution, and reproduction in any medium, provided you give appropriate credit to the original author(s) and the source, provide a link to the Creative Commons license, and indicate if changes were made. The Creative Commons Public Domain Dedication waiver (http://creativecommons.org/publicdomain/zero/1.0/) applies to the data made available in this article, unless otherwise stated. 
disability and functional impairment $[14,15]$. Screening for PAD is therefore advocated in the international guidelines [16]. Comparably, the European Association for the Study of Diabetes recommends annual screening in all patients regardless of risk factors [17]. Of note, PAD is associated with substantial increased risk of fatal and non-fatal cardiovascular and cerebrovascular events, plausibly due to a greater degree of overall systemic atherosclerosis $[18,19]$. Thus, an early detection and timely treatment of clinical risk factors may contribute in reducing the prevalence and severity of PAD in patients with DM and coronary artery disease (CAD).

Nevertheless, the association of PAD with respect to the severity of cardiovascular calcification remains untested, and the impact of DM on development of PAD in patients with CAD needs further study. With the present study, we aimed to investigate the association of cardiovascular calcification with PAD in patients with and without DM undergoing de novo coronary angiography (CAG) in a real-world nationwide setting.

\section{Methods}

\section{Study design and data sources}

All Danish citizens have a unique and permanent civil registration number that enables individual-level linkage across all Danish nationwide registers [20-24]. In the present study, patients were identified by using the Danish Heart Registry (DHR), a clinical database containing information on all patients referred for CAG within the Danish healthcare system [25]. The Danish National Patient Registry holds information on all inpatient and out-patient treatments [recorded as International Classification of Diseases (ICD) codes] nationwide since 1978 and was used to retrieve information on comorbidities, prior to the CAG procedure date [20]. The Danish Register of Medicinal Product Statistics (National Prescription Register) holds information on all prescribed medicine dispensed since 1995 according to The International Anatomical Therapeutic Chemical (ATC) classification system and was used to obtain data on concomitant pharmacotherapy. These Danish registers have previously been shown to be complete and accurate [20,21]. The present study is conducted and reported in accordance with the recommendation of Strengthening the Reporting of Observational Studies in Epidemiology (STROBE) [20].

\section{Inclusion criteria and classification of patients}

This present study included all Danish citizens aged $\geq 18$ years, undergoing first-time CAG over a period of 12 years spanning January 1, 2000 to December 31, 2012. Patients with more than one CAG within the study period were only included at their first appearance only. Moreover, the study subjects were censored on death, migration, and at the end of study period
(December 31, 2012). The study population was then segregated into patients with DM and patients without DM (non-DM) with a further subdivision into five categories of CAD i.e. no-vessel disease, single-vessel disease, double-vessel disease, triple-vessel disease, and diffuse disease. Obstructive coronary artery disease (i.e. single-, double- or triple-vessel disease) was defined as one or more epicardial coronary arteries with $>50 \%$ angiographic lumen narrowing, whereas no-vessel disease was defined as angiographic lumen narrowing $(<50 \%)$ in a single coronary vessel. On the similar lines, patients registered with diffuse (coronary artery) disease includes those with diffuse non-significant atherosclerosis in more than one coronary artery or with non-obstructive $(<50 \%)$ lesions in multiple coronary vessels. Of notice, the degree of vessel disease was to the discretion of the invasive cardiologist.

\section{Outcome}

PAD was the outcome of interest and was defined as all arterial diseases except the atherosclerotic disease of coronary arteries, aorta, and intracranial arteries. ICD-10 diagnoses codes (DI170, DI73, DI74) were used to identify patients with PAD. Moreover, the diagnoses of PAD registered in Danish National Patient Registry are validated with an over-all positive predictive value of $100 \%$ (CI 92.9-100) [26]. Patients with a history of PAD $(n=$ 7.342) at baseline were excluded from the study cohort before the study start.

\section{Pharmacotherapy and comorbidity}

Baseline pharmacotherapy was defined by dispensed prescriptions 180 days prior to CAG. Comorbidities were established based on diagnostic codes recorded in the National Patient Registry, including atrial fibrillation, hypertension, vascular disease, renal disease, and thromboembolism. Hypertension was identified by either a hospital diagnosis for hypertension, or concurrent use of at least 2 of the following classes of antihypertensive agents within a 3-month period: $\alpha$-adrenergic blockers, non-loop diuretics, vasodilators, $\beta$-blockers, calciumchannel blockers, and renin-angiotensin system inhibitors. DHR records information on DM status, body mass index (BMI), and smoking status on all patients undergoing CAG. To increase the sensitivity of the exposure group, we defined DM by use of glucose-lowering agents and a diagnosis of DM retrieved from DHR and National Prescription Register, respectively. The respective ICD, ATC, codes for all examined comorbidities and concomitant medications are presented in Table 1.

\section{Statistical analysis}

Baseline characteristics were presented as medians, frequencies and percentages. $x^{2}$-test was used to test the difference between categorical variables and $t$-test or the 
Table 1 Overview of ICD, and ATC codes for all examined comorbidities and concomitant medications

\begin{tabular}{ll}
\hline $\begin{array}{l}\text { Pharmacological Treatments } \\
\text { Cholesterol-lowering drugs }\end{array}$ & ATC \\
Acetylsalicylic acid & B01AC06 \\
Glucose-lowering agents & A10 \\
Vitamin K antagonists & B01AA \\
Digoxin & C01AA \\
Platelet inhibitors & B01AC \\
Hypertension & \\
A-adrenergic blockers & C02A, C02B, C02C, C02DA, C02L, \\
Non-loop diuretics & C03A, C03B, C03D, C03E, C03X, \\
Vasodilators & C09XA52, C02DB, C02DD, C02DG, \\
B-blockers & C07, C07F, C08, C09BB, C09. \\
Calcium channel blockers & \\
Renin angiotension system & \\
inhibitors & \\
Comorbidity & ICD-10 \\
Vascular disease & I21 to I22 \\
Atrial fibrillation & 148 \\
Thromboembolism & 126, 163, 164, 174, G458, G459 \\
Hypertension & 110 to 115 \\
Renal Disease & N03, N04, N17 to N19, R34, 112, 113 \\
\hline & \\
&
\end{tabular}

Kruskal-Wallis test for differences between continuous variables. The level of statistical significance was set as $p<0.05$. Risk of PAD in 5-years of follow-up, between the respective subgroups were presented as cumulative incidence curves with 95\% confidence interval (CI) using the Aalen-Johansen method. Risk-time was constituted of time since CAG procedure date until an outcome of PAD occurred. Multivariable Cox proportional-hazard models adjusted for sex, age, smoking status, BMI, hypertension, and concomitant medication (beta-blockers, vitamin $\mathrm{K}$ antagonist, platelet inhibitors, acetylsalicylic acid, and cholesterol lowering drugs), were used to estimate hazard ratios (HR) and confidence intervals of PAD between subgroups of patients with and without DM. Patients with no-DM and no-vessel disease were used as references.

All statistical analyses were performed with SAS statistical software version 9.4 (SAS Institute Inc., Cary, North Carolina, USA), $\mathrm{R}$ statistics ( $\mathrm{R}$ Core Team, 2016), and Stata software version 14 (Statacorp, College St., Texas, USA).

\section{Results}

A total of 116,491 patients underwent first-time CAG between January 1, 2000 and December 31, 2012. Patients with previous known PAD by the start of the study $(n=7.342)$ were excluded. A total of 23,969
(20.58\%) were identified with DM. Mean age was 64.31 [IQR $56-73$ years], and $63.67 \%$ of the identified patients were male. A flowchart of the study population is illustrated in Fig. 1, and baseline characteristics stratified by the degree of CAD among patients with and without DM are presented in Table 2.

Table 3 demonstrates number of patients (given in percentages) with DM and non-DM in categories of CAD showing a higher percentage of double, triple, and diffuse-vessel disease among patients with DM, compared to non-DM patients.

The primary endpoint i.e. PAD was diagnosed in 4.737 patients $(4.07 \%)$ in a 5 -year follow-up period with a higher percentage among patients with DM (6.15\%) compared to non-DM patients (3.53\%).

The 5-year cumulative incidence of PAD among DM patients were 8.8, 8.2, 10.2, 13.0, and 6.8\% in no-vessel disease, single-vessel disease, double-vessel disease, triplevessel disease, and diffuse disease, respectively. Whereas, the corresponding cumulative incidence of PAD among patients with non-DM were 4.9, 4.8, 6.0, 8.4, and 6.1\% in novessel disease, single-vessel disease, double-vessel disease, triple-vessel disease, diffuse disease, respectively (Fig. 2).

Correspondingly, the age- and sex-adjusted cox regression models yielded increased hazards of PAD among DM patients with HR 1.73 (1.59-1.87), 1.91 (1.63-2.24), 2.37 (1.99-2.83), 2.94 (2.57-3.37), and 1.59 (1.28-1.97) in no-vessel disease, single-vessel disease, double-vessel disease, triple-vessel disease, and diffuse disease, respectively. On the contrary, hazards of PAD in non-DM patients were comparably lower with HR 1.0, 1.12 (0.92-1.05), 1.30 (1.17-1.36), 1.77 (1.53-1.72), and $1.36(0.92-1.06)$ in novessel disease, single-vessel disease, double-vessel disease, triple-vessel disease, diffuse disease, respectively. $\mathrm{P}$ for interaction between $\mathrm{DM}$ and degree of CAD, compared to nonDM was 0.042. Furthermore, the fully adjusted HRs remained significant, showing an increased risk of PAD in patients with $\mathrm{DM}$ that appears to increase with the severity of CAD (Table 4).

\section{Discussion}

In a nationwide register-based cohort study on all patients undergoing CAG, DM was observed to be independently associated with increasing risk of PAD dependent on severity of CAD. Moreover, the over-all associated risk of PAD remained significantly increased in patients with DM compared to non-DM patients.

PAD is defined as atherosclerosis of the lower extremity arteries and it is associated with increased risk of lower extremity amputation and impaired life quality [27]. Studies have linked this group of patients with a higher risk of DM [8-10, 28]. DM-associated atherosclerosis affects all major vascular beds, including cardiovascular system and arteries of the lower extremity 


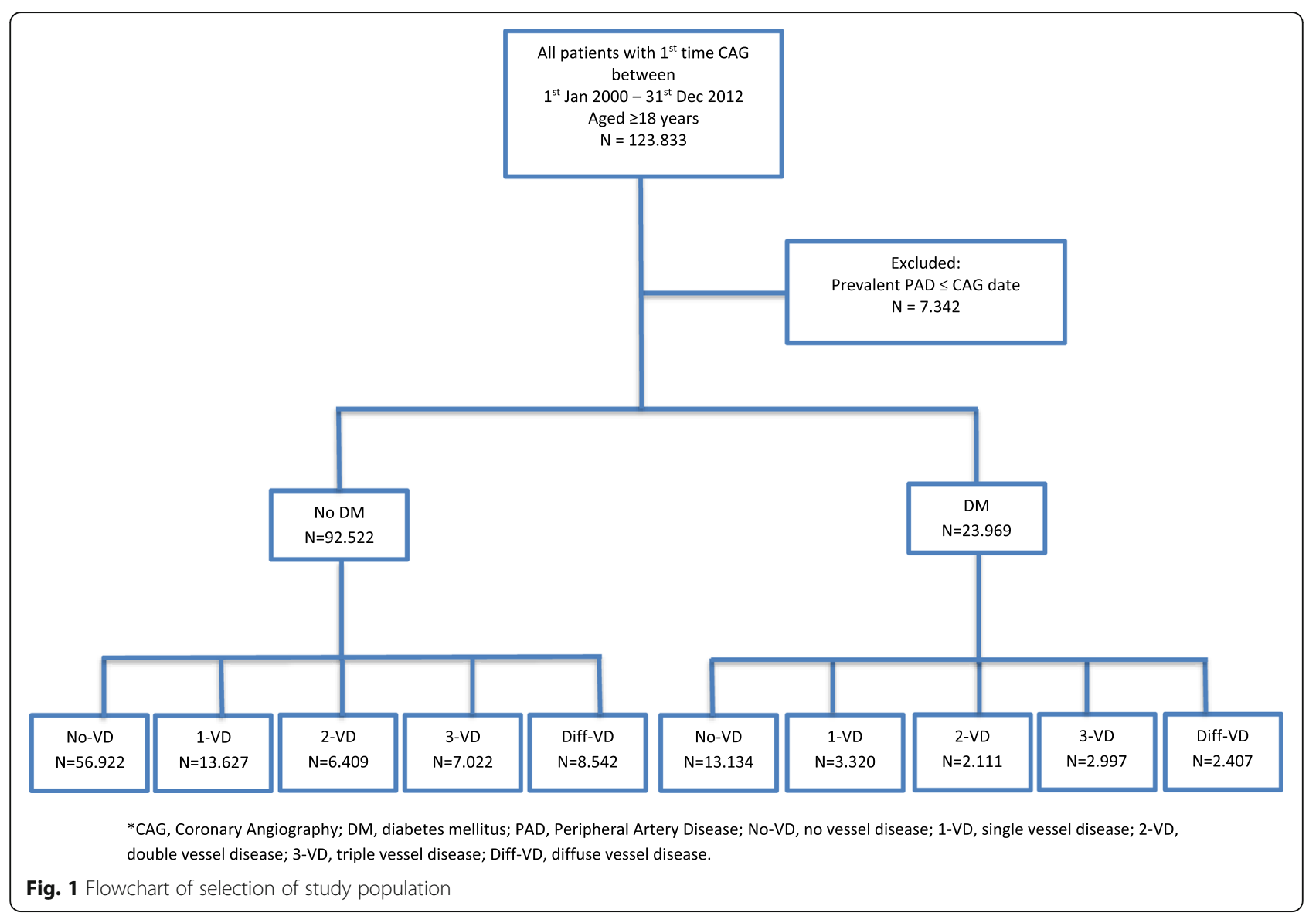

and thereby contributes to cardiovascular disease burden and mortality [29]. An estimated $15 \%$ of patients with DM are reported to have PAD within 10 years after the time they are diagnosed with DM, and the number increases to $45 \%$ after 20 years [19]. PAD in patients with DM causes long-term disability including ischemic ulcer or gangrene, which ultimately may result in limb amputations [30]. The American Diabetes Association consensus panel recommends screening for PAD in all DM patients above the age of 50 , or if they have risk factors such as smoking, dyslipidemia, and hypertension [16].

Although some studies have addressed this subject, results are based on limited data [18, 31, 32] and larger population based studies are warranted. We, therefore, used Danish national registers to examine the risk of PAD in patients with DM compared to non-diabetics and its relationship to CAD in a nationwide setting. The results in the present study confirm previous observations of an augmented risk of developing PAD in patients with DM and CAD, compared to patients without DM [31, 32]. Furthermore, this risk increases by the severity of CAD in patients with and without DM. However, in our study patients with diffuse disease in DM patients appears to have a lower cumulative incidence of PAD compared to no-vessel disease (Fig. 2). As the cumulative incidences of an event of interest are calculated in the presence of a competing risk (e.g. death), this variance in diffuse disease group could be explained by the substantial number of patients that die during the follow-up without experiencing the study outcome. Moreover, the HRs of PAD in patients with DM who suffer diffuse disease compared to no-vessel disease are not significantly different. Also, compared to diffuse and obstructive coronary artery disease, the proportion of patients with no-vessel disease was found to be higher in patients with DM (54\%) and non-DM (63\%). The degree of vessel disease recorded in DHR was subject to the discretion of the invasive cardiologist, which could result in more patients with borderline angiographic lumen narrowing (i.e. slightly higher than $50 \%$ ) that are most likely registered as no-vessel disease. Of notice, patients at risk of developing CAD (including patients with DM) are most likely to be referred at earlier course of their disease, which may also explain a higher proportion of diffuse, no-vessel or single-vessel disease. Indeed, this notion is supported by the results demonstrated by $\mathrm{Da}$ nish Health Authority (Sundhedsstyrelsen) and Danish Heart Foundation (Hjerteforeningen) indicating an increasing trend in number of coronary angioplasties per million Danish inhabitants [33]. 


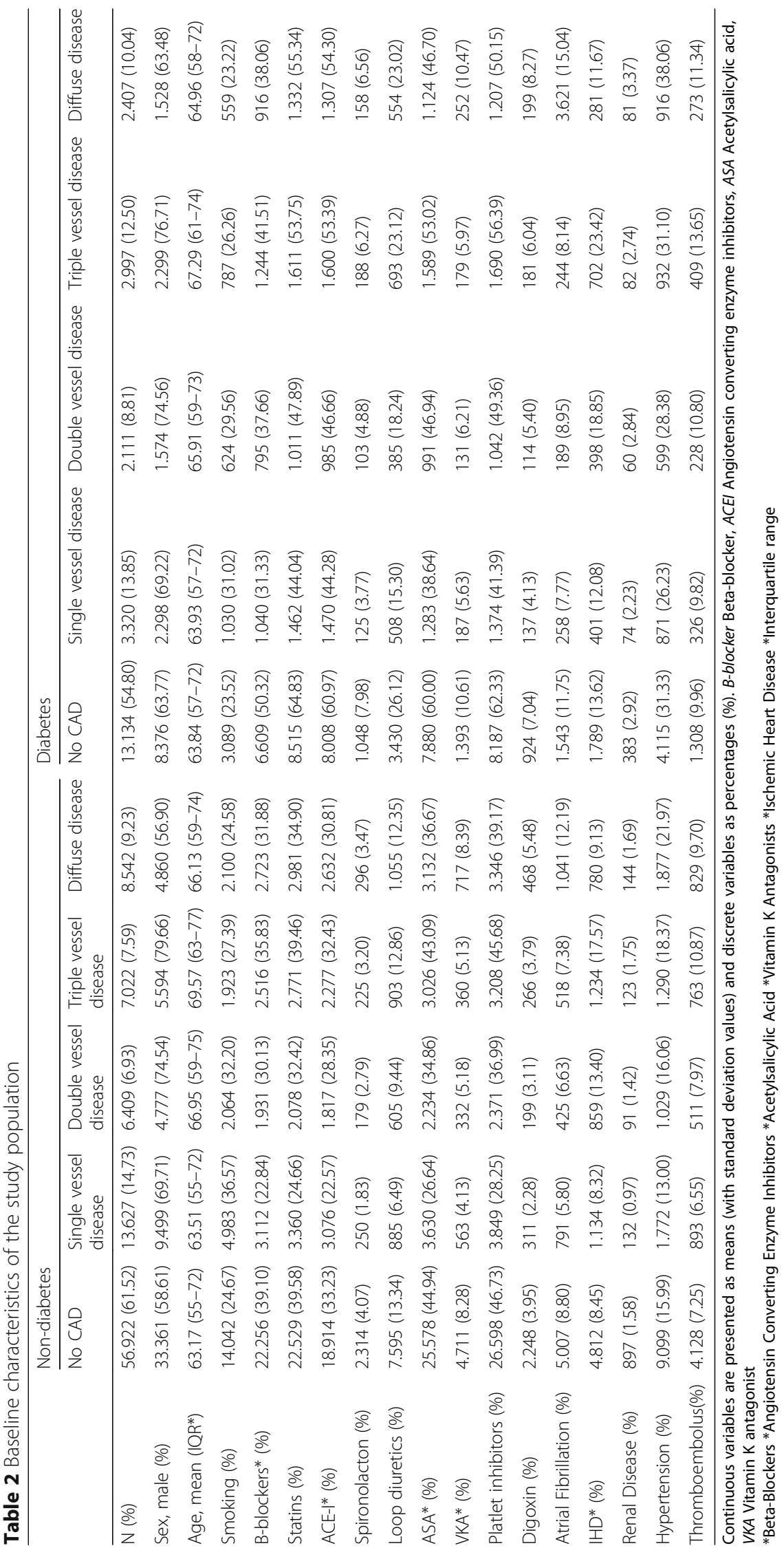


Table 3 Percentages of CAD in patients with and without DM

\begin{tabular}{lll}
\hline Degree of CAD & Non-DM & DM \\
\hline No vessel disease & $61.52 \%$ & $54.80 \%$ \\
Single vessel disease & $14.73 \%$ & $13.85 \%$ \\
Double vessel disease & $6.93 \%$ & $8.81 \%$ \\
Triple vessel disease & $7.59 \%$ & $12.50 \%$ \\
Diffuse vessel disease & $9.23 \%$ & $10.04 \%$ \\
\hline
\end{tabular}

DM is characterized by hyperglycemia, insulin resistance, and dyslipidemia that contribute to development and progression of PAD, through pathophysiological mechanisms (e.g. vascular inflammation, endothelial dysfunction, hemostasis dysregulation) similar to those in CAD $[12,34]$. Vascular inflammation is a risk marker of atherothrombosis, and studies have shown that CAD patients with an additional diagnosis of PAD have higher circulating levels of inflammatory biomarkers [9, 35]. In view of that, the vascular inflammation caused by DM may play a contributing role in development of PAD by accelerating the disease process in patients with known CAD [12]. In addition, choice of glucose-lowering agents
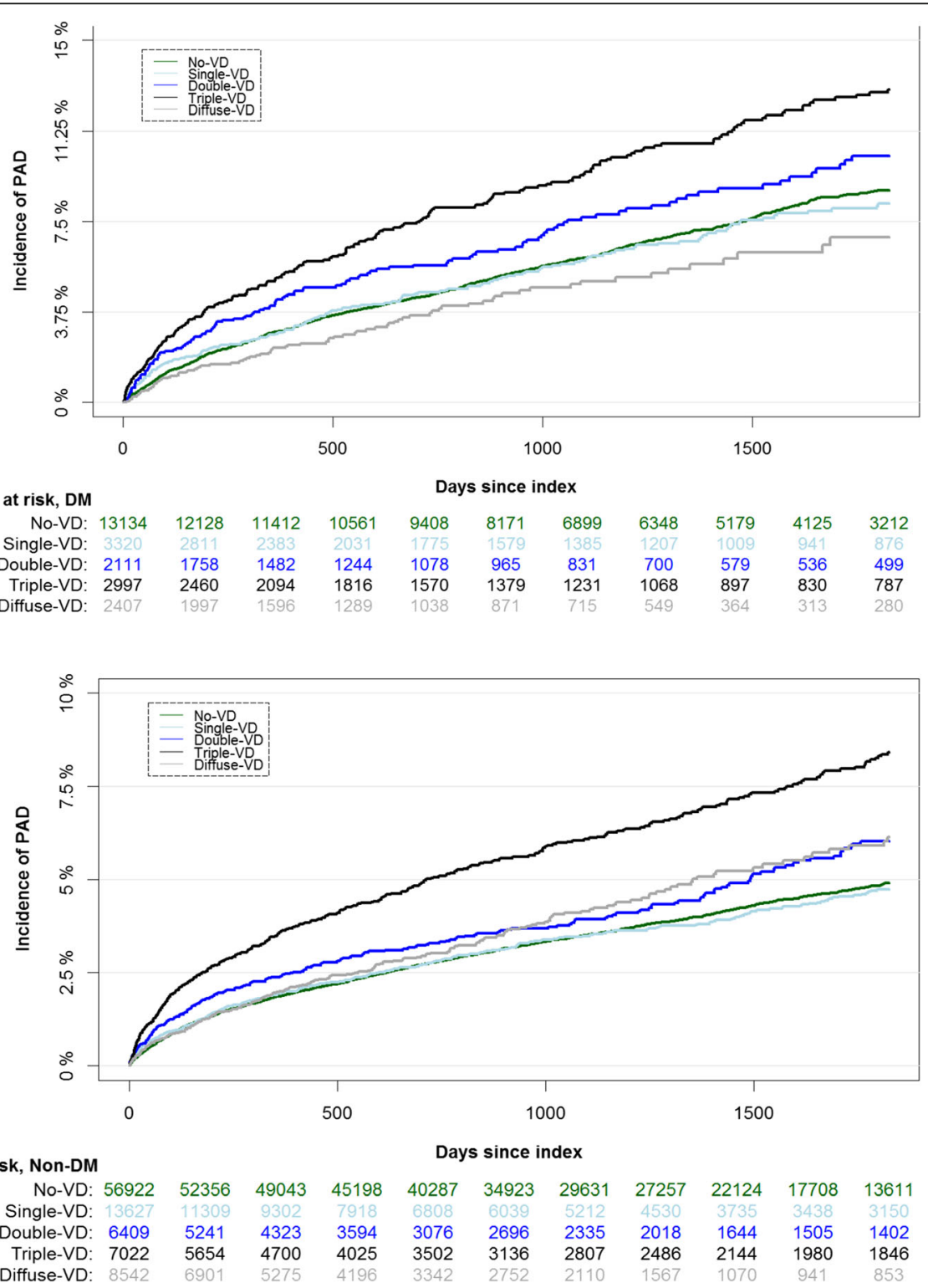

Fig. 2 Cumulative Incidence Curve and patients at risk within 5 years from coronary angiography stratified by degree of CAD in patients with DM compared to non-DM patients. At risk table represents number of patients at risk of event at given time 
Table 4 Hazard ratios and 95\% confidence intervals of PAD in patients with and without DM with respect to degree of CAD along with individual covariates

\begin{tabular}{llll}
\hline & Hazard Ratio & $\begin{array}{l}\text { 95\% Confidence } \\
\text { Interval }\end{array}$ & P-Value \\
\hline Non-DM & & NA & NA \\
No-CAD (reference) & 1.0 & $1.03-1.29$ & 0.013 \\
Single-vessel disease & 1.15 & $1.18-1.58$ & $<0.001$ \\
Double-vessel disease & 1.37 & $1.61-2.05$ & $<0.001$ \\
Triple-vessel disease & 1.82 & $1.16-1.52$ & $<0.001$ \\
Diffuse-vessel disease & 1.33 & & \\
DM & & $1.55-1.86$ & $<0.001$ \\
No-CAD & 1.70 & $1.67-2.31$ & $<0.001$ \\
Single-vessel disease & 1.96 & $1.96-2.82$ & $<0.001$ \\
Double-vessel disease & 2.35 & $2.50-3.30$ & $<0.001$ \\
Triple-vessel disease & 2.87 & $1.17-1.84$ & $<0.001$ \\
Diffuse-vessel disease & 1.46 & & \\
\hline
\end{tabular}

may have an important impact on the development of atherosclerotic plaques [36]. Moreover, risk of developing PAD in patients with concurrent DM and advance CAD also depend on interplay between factors such as comorbidities, smoking, obesity, and reduced physical activity [11]. These factors foster development of atherothrombosis and they are often present before the diagnosis of DM and worsen in line with the duration of DM [37]. Moreover, due to the distal territory of vessel involvement and its association with peripheral neuropathy, PAD is more commonly asymptomatic in patients with DM and may present later at a more advanced stage [38]. Also, the lower resistance to infection of this group dramatically increases their risk of amputation compared to non-diabetic patients with PAD [30]. Thus, an early detection and timely treatment of clinical risk factors may contribute in reducing the prevalence and severity of PAD in patients with DM and CAD.

Taken together, results from the present study add to the existing evidence that patients with concurrent DM and $C A D$ may be at increased risk of developing PAD and this risk appears to worsen in advance CAD. Clinicians should be aware and may want to consider screening and treatment at early stage to elicit the adverse outcomes, specifically in high risk population of patients with DM and CAD. Nevertheless, prospective studies aimed to explore effects of such outcomes are required.

\section{Strengths and limitations}

Large number of unselected patients in a real-world nationwide setting, completeness of follow-up, and use of validated measures of exposure and outcome are among the strengths of the present study. Furthermore, healthcare in Denmark is equally accessible to all Danish citizens minimizing confounding by variables associated with social class. It is our opinion that this large-scale nationwide study provides a reliable image of the CAG population under investigation.

There are also several limitations in the study that must be acknowledged. The observational nature of the study only allows to establish association and does not represent cause-and-effect relationships. Moreover, the subpopulation was identified by using diagnosis from registers along with data on claimed prescriptions. Although the registration of data in Danish registers is thought to be accurate, there might be some limitations regarding the PAD diagnoses; especially as PAD is underreported due to the often asymptomatic nature of the disease. Also, development of neuropathy in patients with DM often suppresses PAD symptoms leading to late diagnosis of PAD. In opposite, patients with DM are followed closely with regularly health checkups that might lead to earlier detection of PAD. The registries used in this study do not include information on glycosylated hemoglobin levels and patients with DM managed with diet alone or developing DM during the follow up period were not identified. To address this issue, we defined DM by use of glucose lowering agents and the recorded diagnoses of DM in DHR and National Prescription Register, respectively. Nevertheless, the results are only valid for patients with DM requiring pharmacotherapy at the time of CAG. Moreover, DHR lacked information on various clinical parameters including thorough data on the luminal diameter and precise extent of coronary artery stenosis (in percentage).

\section{Conclusion}

In this observational nationwide study of patients undergoing CAG, DM was associated with increased risk of PAD in patients with and without established CAD. Additionally, we observed increasing risk of PAD with more extensive CAD. Further studies are warranted to support these findings.

\section{Abbreviations \\ Acknowledgements \\ Not applicable.}

BMI: Body mass index; CAD: Coronary artery disease; CAG: Coronary angiography; DM: Type 2 diabetes mellitus; HR: Hazard ratio; IR: Incidence rate; PAD: Peripheral artery disease

\section{Authors' contributions}

SK and GG designed the study and performed statistical analyses. SK wrote the initial draft of the paper. All authors (SK, TS, NC, KH, JFL, CNB, HD, CTP, GG) contributed equally to interpretation of data, revised the paper for important intellectual content and approved the final version of the paper.

\section{Funding}

The study was financially supported by "Herlev/Gentofte Intern Forskningspulje". The study sponsors had no influence on data collection, interpretation of data, nor the final decision to submit the manuscript for publication. 


\section{Availability of data and materials}

The datasets generated and/or analysed during the current study are not publicly available but are accessible on reasonable request and through permission from DST (Danmark statistik).

\section{Ethics approval and consent to participate}

The study was approved by the Danish Data Protection Agency (ref. 200758-0015, int. ref.: GEH-2014-018), and data were made available in a way that individuals could not be identified. Approval from an ethics committee is not required for retrospective register-based studies in Denmark. The data that support the findings of this study are available from third party (DST, Danmark Statistik) but restrictions apply to the availability of these data and they are not publicly available. Data are only available upon reasonable request and through permission from DST.

\section{Consent for publication}

Not applicable.

\section{Competing interests}

The authors declare that they have no competing interests.

\section{Author details}

'Department of Cardiology, Copenhagen University Hospital Herlev-Gentofte, Herlev and Gentofte Hospital, Kildegaardsvej 28, 2900 Hellerup, Denmark. ${ }^{2}$ Department of Cardiology, Bispebjerg-Frederiksberg University Hospital, Copenhagen, Denmark. ${ }^{3}$ Department of Biomedical Sciences, University of Copenhagen, Copenhagen, Denmark. ${ }^{4}$ Department of Nephrology, Copenhagen University Hospital Rigshospitalet, Copenhagen, Denmark. ${ }^{5}$ The Danish Heart Foundation, Copenhagen, Denmark. ${ }^{6}$ Department of Vascular Surgery, Kolding Hospital, Kolding, Denmark. Department of Regional Health Research, University of Southern Denmark, Odense, Denmark. ${ }^{8}$ Department of Cardiology, Odense University Hospital, Odense, Denmark. ${ }^{9}$ Department of Cardiology, Copenhagen University Hospital Rigshospitalet, Copenhagen, Denmark. ${ }^{10}$ Department of Cardiology, Nordsjaellands Hospital, Hilleroed, Denmark.

Received: 26 March 2019 Accepted: 30 September 2019

Published online: 24 October 2019

\section{References}

1. Schorr EN, Treat-Jacobson D, Lindquist R. The relationship between peripheral artery disease symptomatology and ischemia. Nurs Res. 2017; 66(5):378-87.

2. Ciocan RA, Bolboaca SD, Radulescu S, Stancu B, Ciocan A, Gherman CD. Demographic and comorbidity pattern of patients with critical limb ischemia. Folia Med. 2017:59(1):14-22.

3. Davidson MH. Cardiovascular risk factors in a patient with diabetes mellitus and coronary artery disease: therapeutic approaches to improve outcomes: perspectives of a preventive cardiologist. Am J Cardiol. 2012;110(9 Suppl): $43 b-9 b$.

4. Criqui MH, Langer RD, Fronek A, Feigelson HS, Klauber MR, McCann TJ, et al. Mortality over a period of 10 years in patients with peripheral arterial disease. N Engl J Med. 1992;326(6):381-6.

5. Inglis SC, Hermis A, Shehab S, Newton PJ, Lal S, Davidson PM. Peripheral arterial disease and chronic heart failure: a dangerous mix. Heart Fail Rev. 2013;18(4):457-64.

6. Paraskevas Kl, Mukherjee D, Whayne TF Jr. Peripheral arterial disease: implications beyond the peripheral circulation. Angiology. 2013;64(8):569-71.

7. Marso SP, Hiatt WR. Peripheral arterial disease in patients with diabetes. J Am Coll Cardiol. 2006;47(5):921-9.

8. Ali Z, Ahmed SM, Bhutto AR, Chaudhry A, Munir SM. Peripheral artery disease in type II diabetes. J Coll Physicians Surg Pak. 2012;22(11):686-9.

9. Snell-Bergeon JK, Budoff MJ, Hokanson JE. Vascular calcification in diabetes: mechanisms and implications. Curr Diab Rep. 2013;13(3):391-402.

10. Jude EB, Eleftheriadou I, Tentolouris N. Peripheral arterial disease in diabetes--a review. Diabet Med. 2010;27(1):4-14.

11. Joosten MM, Pai JK, Bertoia ML, Rimm EB, Spiegelman D, Mittleman MA, et al. Associations between conventional cardiovascular risk factors and risk of peripheral artery disease in men. JAMA. 2012;308(16):1660-7.
12. van Ark J, Moser J, Lexis CP, Bekkema F, Pop I, van der Horst IC, et al. Type 2 diabetes mellitus is associated with an imbalance in circulating endothelial and smooth muscle progenitor cell numbers. Diabetologia. 2012;55(9):2501-12.

13. Mercer BN, Morais S, Cubbon RM, Kearney MT. Diabetes mellitus and the heart. Int J Clin Pract. 2012;66(7):640-7.

14. Argyriou C, Saleptsis V, Koutsias S, Giannoukas AD. Peripheral arterial disease is prevalent but underdiagnosed and undertreated in the primary care setting in Central Greece. Angiology. 2013;64(2):119-24.

15. Faglia E. Characteristics of peripheral arterial disease and its relevance to the diabetic population. Int J Low Extrem Wounds. 2011;10(3):152-66.

16. Bosevski M. Peripheral arterial disease and diabetes. Prilozi. 2012;33(1):65-78.

17. Standards of medical care in diabetes. Diabetes Care. 2005;28(Suppl 1):S4-s36. https://doi.org/10.2337/diacare.28.suppl_1.S4.

18. Sukhija R, Yalamanchili K, Aronow WS. Prevalence of left main coronary artery disease, of three- or four-vessel coronary artery disease, and of obstructive coronary artery disease in patients with and without peripheral arterial disease undergoing coronary angiography for suspected coronary artery disease. Am J Cardiol. 2003;92(3):304-5.

19. Yilmaz MB, Guray Y, Guray U, Biyikoglu SF, Tandogan I, Korkmaz S. Metabolic syndrome increases the risk of significant coronary artery involvement in patients with peripheral artery disease. Coron Artery Dis. 2006;17(6):529-32.

20. Lynge E, Sandegaard JL, Rebolj M. The Danish National Patient Register. Scand J Public Health. 2011;39(7 Suppl):30-3.

21. Gaist D, Sorensen HT, Hallas J. The Danish prescription registries. Dan Med Bull. 1997;44(4):445-8.

22. Schmidt M, Pedersen $L$, Sorensen $H T$. The Danish civil registration system as a tool in epidemiology. Eur J Epidemiol. 2014;29(8):541-9.

23. Thygesen LC, Daasnes C, Thaulow I, Bronnum-Hansen H. Introduction to Danish (nationwide) registers on health and social issues: structure, access, legislation, and archiving. Scand J Public Health. 2011;39(7 Suppl):12-6.

24. von Elm E, Altman DG, Egger M, Pocock SJ, Gotzsche PC, Vandenbroucke $J P$. The strengthening the reporting of observational studies in epidemiology (STROBE) statement: guidelines for reporting observational studies. J Clin Epidemiol. 2008;61(4):344-9.

25. Schmidt M, Maeng M, Madsen M, Sorensen HT, Jensen LO, Jakobsen CJ. The Western Denmark heart registry: its influence on cardiovascular patient care. J Am Coll Cardiol. 2018;71(11):1259-72.

26. Thygesen SK, Christiansen CF, Christensen S, Lash TL, Sorensen HT. The predictive value of ICD-10 diagnostic coding used to assess Charlson comorbidity index conditions in the population-based Danish National Registry of patients. BMC Med Res Methodol. 2011;11:83.

27. Hirsch AT, Haskal ZJ, Hertzer NR, Bakal CW, Creager MA, Halperin JL, et al. ACC/AHA 2005 PRACTICE Guidelines for the management of patients with peripheral arterial disease (lower extremity, renal, mesenteric, and abdominal aortic): a collaborative report from the American Association for Vascular Surgery/Society for Vascular Surgery, Society for Cardiovascular Angiography and Interventions, Society for Vascular Medicine and Biology, Society of Interventional Radiology, and the ACC/AHA Task Force on Practice Guidelines (Writing Committee to Develop Guidelines for the Management of Patients With Peripheral Arterial Disease): endorsed by the American Association of Cardiovascular and Pulmonary Rehabilitation; National Heart, Lung, and Blood Institute; Society for Vascular Nursing; TransAtlantic Inter-Society Consensus; and Vascular Disease Foundation. Circulation. 2006;113(11):e463-654.

28. Rhee SY, Kim YS. Peripheral arterial disease in patients with type 2 diabetes mellitus. Diabetes Metab J. 2015;39(4):283-90.

29. Cornejo Del Rio V, Mostaza J, Lahoz C, Sanchez-Arroyo V, Sabin C, Lopez S, et al. Prevalence of peripheral artery disease (PAD) and factors associated: an epidemiological analysis from the population-based screening PRE-diabetes and type 2 DIAbetes (SPREDIA-2) study. PLoS One. 2017;12(10):e0186220.

30. Gurney JK, Stanley J, York S, Rosenbaum D, Sarfati D. Risk of lower limb amputation in a national prevalent cohort of patients with diabetes. Diabetologia. 2018;61(3):626-35.

31. Agarwal AK, Singh M, Arya V, Garg U, Singh VP, Jain V. Prevalence of peripheral arterial disease in type 2 diabetes mellitus and its correlation with coronary artery disease and its risk factors. J Assoc Physicians India. 2012;60:28-32

32. Sarangi S, Srikant B, Rao DV, Joshi L, Usha G. Correlation between peripheral arterial disease and coronary artery disease using ankle brachial index-a study in Indian population. Indian Heart J. 2012;64(1):2-6.

33. Available from: http://www.sst.dk/ /media/DBA260D59A054685A46916 97D622C7D1.ashx 
34. Sardu C, Barbieri M, Balestrieri ML, Siniscalchi M, Paolisso P, Calabro P, et al. Thrombus aspiration in hyperglycemic ST-elevation myocardial infarction (STEMI) patients: clinical outcomes at 1-year follow-up. Cardiovasc Diabetol. 2018:17(1):152.

35. Landsberg L, Molitch M. Diabetes and hypertension: pathogenesis, prevention and treatment. Clin Exp Hypertens (New York, NY : 1993). 2004;26(7-8):621-8

36. Balestrieri ML, Rizzo MR, Barbieri M, Paolisso P, D'Onofrio N, Giovane A, et al. Sirtuin 6 expression and inflammatory activity in diabetic atherosclerotic plaques: effects of incretin treatment. Diabetes. 2015;64(4):1395-406.

37. Vrsalovic M, Vucur K, Vrsalovic Presecki A, Fabijanic D, Milosevic M. Impact of diabetes on mortality in peripheral artery disease: a meta-analysis. Clin Cardiol. 2017:40(5):287-91.

38. Freisinger $\mathrm{E}$, Malyar NM, Reinecke H, Lawall H. Impact of diabetes on outcome in critical limb ischemia with tissue loss: a large-scaled routine data analysis. Cardiovasc Diabetol. 2017;16(1):41.

\section{Publisher's Note}

Springer Nature remains neutral with regard to jurisdictional claims in published maps and institutional affiliations.

Ready to submit your research? Choose BMC and benefit from:

- fast, convenient online submission

- thorough peer review by experienced researchers in your field

- rapid publication on acceptance

- support for research data, including large and complex data types

- gold Open Access which fosters wider collaboration and increased citations

- maximum visibility for your research: over $100 \mathrm{M}$ website views per year

At BMC, research is always in progress.

Learn more biomedcentral.com/submissions 